\title{
Development and utility of pirfenidone in the treatment of idiopathic pulmonary fibrosis: review of preclinical science and recent clinical trials
}

This article was published in the following Dove Press journal:

Transplant Research and Risk Management

9 April 2011

Number of times this article has been viewed

\section{Robert M Jackson Orlando Gomez-Marin}

Research Service, Miami Veterans Administration Healthcare System (VAHS), Miami, FL, USA
Correspondence: $\mathrm{R}$ Jackson

Research Service (I5I), VAHS, I20I NW

16th Street, Miami, FL 33125, USA

$\mathrm{Tel}+\mathrm{I} 3055753548$

Fax + I 3055753 I 26

Email ipf@med.miami.edu

\begin{abstract}
Pirfenidone is a pyridine-derived, double-ringed molecule that has a number of biologic effects including anti-inflammatory and antifibrotic properties in rodent models of acute lung injury and fibrosis, in addition to scavenging reactive oxygen species. These effects are clinically relevant, because idiopathic pulmonary fibrosis (IPF) is a progressive and increasingly prevalent disease characterized by diffuse lung scarring due to alveolar epithelial cell injury, which typically leads to death 3-5 years after diagnosis. No proven therapy for IPF exists, and many IPF patients eventually require lung transplantation, making the need for pharmacologic therapy great. Pirfenidone is rapidly distributed in body water and metabolized by the liver. Several clinical trials have tested pirfenidone in patients with IPF and found it well-tolerated with acceptable side effects. Pirfenidone in clinical trials has been found to improve progression-free survival and pulmonary function of IPF patients. Although the specific mechanism accounting for its benefits is not known, pirfenidone decreases collagen synthesis and fibroblast proliferation, and it may down-regulate inflammation by virtue of its effects on mitogen-activated protein kinases. Pirfenidone has gained regulatory approval for marketing in Japan and in the European Union. It could prove to be a useful therapeutic agent for patients with IPF.

Keywords: idiopathic pulmonary fibrosis, pirfenidone, redox signaling
\end{abstract}

\section{Introduction}

Pirfenidone has been found over the last three decades to reduce inflammation, limit fibrosis, and have radical scavenging properties, which have made it interesting both to scientists investigating basic mechanisms of fibrosis and clinicians seeking therapies for fibrotic diseases. ${ }^{1}$ Pirfenidone has enjoyed recent interest because of antifibrotic properties in the lung and its testing in large scale clinical trials. ${ }^{2}$

Idiopathic pulmonary fibrosis (IPF), a fatal fibrosing lung disease occurring predominantly in older men, has been the most prominent target in clinical trials. Smaller trials have been reported in other fibrosing diseases. Therefore, it is timely to review its underlying chemistry, pharmacology, and preclinical testing, which could lead to the use of pirfenidone in several disease states. Pirfenidone's effectiveness in preclinical trials of lung fibrosis has also stimulated interest in its possible therapeutic use in diseases that result in kidney or liver fibrosis, among others.

This review presents an overview of preclinical and clinical studies involving pirfenidone mainly in the context of IPF. We present clinical information that demonstrates a need for effective antifibrotic therapy, and summarize basic chemical 
and pharmacological data that have led to clinical testing of pirfenidone. Finally, we place the future clinical use of pirfenidone in the context of preceding basic and clinical research.

Pirfenidone clearly inhibits bleomycin-induced lung inflammation and fibrosis in rodents. ${ }^{3}$ Cell culture studies have confirmed its inhibitory effects on collagen production induced by transforming growth factor-beta (TGF- $\beta$ ), as described below. ${ }^{4}$

It is useful as a drug, since pirfenidone is rapidly absorbed and reaches peak concentration in the circulation in 30-60 minutes. It is rapidly metabolized by the liver and excreted by the kidney. ${ }^{5}$ It appears well-tolerated in human trials. ${ }^{2}$

Large international clinical trials have tested the efficacy of pirfenidone in IPF. Pirfenidone has been presented to United States and European regulatory authorities for marketing approval, and it may soon prove useful in clinical practice.

\section{Clinical context}

IPF is a progressive disease characterized by diffuse lung scarring, which typically results in death about 3-5 years after diagnosis. ${ }^{6}$ While evidence exists for alveolar epithelial cell injury as a precedent of IPF, the predominant pathophysiology involves dysregulated fibrosis, suggesting aberrant wound healing. Alveolar epithelial cell injury may result in an endoplasmic reticulum stress response that leads to fibrosis. ${ }^{7}$

The epidemiology of IPF is not entirely clear, but around 100,000 cases probably exist in the United States at any time, and the incidence may be around 30,000 new cases per year. ${ }^{8}$

A number of risk factors for IPF have been recognized. An increasing number of cases are defined as familial or genetic. Surfactant protein C (SP-C) and other gene abnormalities may account for a significant fraction of IPF cases; other possible inherited risk factors are being identified. ${ }^{9}$ Cigarette smoke exposure is a major factor that increases the relative risk of developing IPF. Although biologically plausible, causality attributable to smoking has not been established. ${ }^{10}$ Inhalation of wood, heavy metal, or other environmental dusts increases risk of developing IPF. ${ }^{11}$ Aspiration of stomach acid possibly due to gastroesophageal reflux is associated with IPF, although it is not directly related to the pathogenesis of fibrosis. ${ }^{12}$

IPF is one of the idiopathic interstitial pneumonias, a group of diffuse parenchymal lung diseases characterized by combination of inflammation, cellular infiltration, and fibrosis. Among these, it is the most common and clinically significant. IPF usually has a slow, insidious onset, and symptoms may present years or months before diagnosis. The pathological pattern of chronic, fibrosing interstitial pneumonia is characterized histologically as usual interstitial pneumonia (UIP). ${ }^{13}$
UIP is typified by multiple areas of proliferating myofibroblasts (fibroblastic foci), and temporal heterogeneity ranging from alveolar epithelial cell injury to accumulation of mature interstitial collagen. A number of growth factors and cytokines have been implicated in myofibroblast proliferation and collagen production, including TGF- $\beta$, insulin-like growth factor (IGF-1), keratinocyte growth factor (KGF), connective tissue growth factor (CTGF), and platelet-derived growth factor (PDGF). TGF- $\beta$ is generally recognized as a predominant driver of the fibrotic phenotype. ${ }^{14}$

IPF is most readily detected by high resolution computed tomographic (HRCT) scanning of the chest as in Figure 1. Typical findings include bilateral, peripheral reticular abnormalities, which involve the lung bases more than the apices, subpleural honeycombing that becomes more prominent over time, traction bronchiectasis, and thickened interlobular septae. Ground glass opacities are typically minimal. ${ }^{15}$

\section{Antifibrotic therapies}

No proven therapies for IPF exist, although several agents have been in clinical trials. Antifibrotic therapies have been proposed as possibly effective in IPF; however, most have failed when subjected to large-scale clinical trials or extensive clinical experience. Many patients require lung transplantation.

Corticosteroids were one of the earliest treatments used for IPF, because lung fibrosis was seen as final result of long-term inflammation. ${ }^{16-19}$ Misclassification of lung diseases occurring in corticosteroid-responsive conditions, such as collagenvascular diseases, reinforced the use of corticosteroids in these

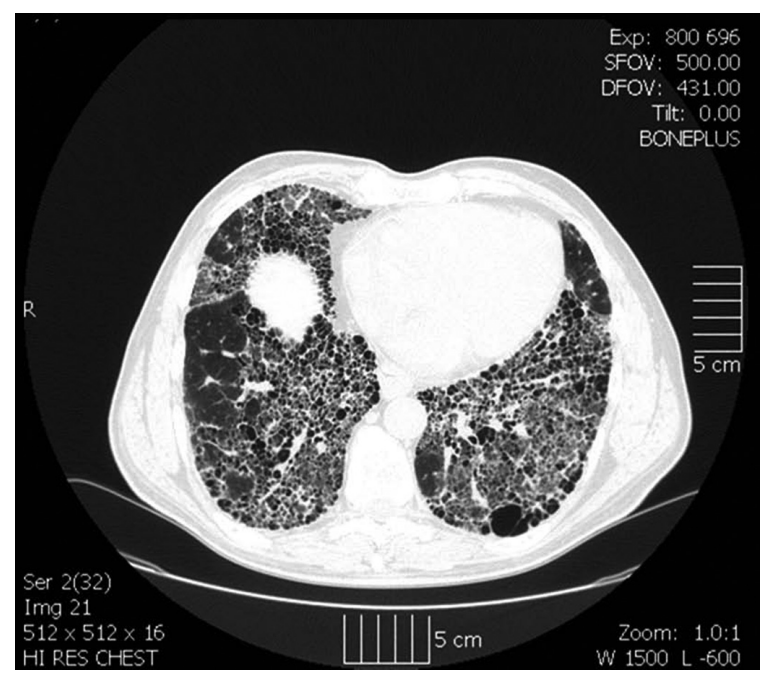

Figure I High resolution computed tomographic (HRCT) scan shows advanced idiopathic pulmonary fibrosis in a patient who eventually required lung transplantation. The scan demonstrates interlobular thickening, traction bronchiectasis and advanced honeycomb formation at the peripheral lung bases. 
studies. Although frequently used until recently, corticosteroids are now recognized as not effective in true IPF, although some patients, apparently with concomitant inflammatory processes, may appear to respond favorably. ${ }^{13}$

Corticosteroids with or without azathioprine continue to be used as treatment for IPF, but no prospective studies showing efficacy of combination anti-inflammatory therapies have been published. ${ }^{20-25}$ Most studies using a combination of corticosteroids and other immunosuppressive agents to treat IPF have failed to show a statistically significant improvement in survival. A multicenter NIH sponsored trial evaluating prednisone and azathioprine in IPF is underway (PANTHER-IPF; NTC 00650091).

No clinically useful biomarkers exist as surrogate endpoints in IPF trials. One study that sampled bronchoalveolar lavage fluid from IPF patients found decreases in neutrophils in the group receiving high-dose corticosteroids compared to those receiving low-dose. ${ }^{26}$ It is unclear in IPF whether biomarkers of inflammation are related to disease progression, so the significance of this finding is unknown.

Pulmonary hypertension commonly develops in patients with IPF. ${ }^{2,13}$ Bosentan, an endothelin antagonist with antifibrotic properties, failed to affect pulmonary function, survival, or exercise tolerance measured by a 6-minute walk in IPF patients. ${ }^{27}$ Similar negative results have been reported using other vasodilators.

Oxidant stress in the alveolar space has been documented in IPF patients and it may be pathophysiologically important in the development of fibrosis. ${ }^{14} \mathrm{~N}$-acetyl-L-cysteine (N-Ac or NAC), a sulfhydryl antioxidant, appeared to suppress progressive decreases in respiratory function of IPF patients who were also treated with azathioprine and prednisone. ${ }^{28}$ That finding suggested the importance of redox signaling in IPF progression. No true control group was studied, so additional data from placebo-controlled trials are required.

Prednisolone (prednisone) and azathioprine along with $\mathrm{N}$-acetyl-L-cysteine carries a weak society recommendation with the stipulation that further studies, such as that cited above, are required. ${ }^{13}$

Therefore, while many agents including pirfenidone have been tested in IPF patients, none have been found that unequivocally improve outcomes in IPF. ${ }^{29,30}$ Supportive care is considered an important and specific treatment strategy in all patients with IPF. ${ }^{13}$

\section{Chemical and molecular properties}

Pirfenidone is a heterocyclic pyridone, the molecular weight of which is 185.22 grams/mole. The International Union for Physics and Applied Chemistry name for pirfenidone is 5-methyl-1-phenyl-1H-pyridin-2-one; ${ }^{31}$ however, alternate nomenclature often appears in the literature. The molecular structure of pirfenidone is shown in Figure 2. Pyridines are derived from coal carbonization and can be synthesized from acetaldehyde and ammonia. Pyridines have industrial uses as solvents, denaturants, and dyes. The compound is two-fold more soluble $(10 \mathrm{mg} / \mathrm{mL})$ in dimethyl sulfoxide than in water.

Pirfenidone appears to possess reactive oxygen species (ROS) scavenging properties, independent of its antifibrotic effects. Pirfenidone inhibits NADPH-dependant lipid peroxidation in a dose-dependant fashion in vitro. ${ }^{32}$ It does not scavenge superoxide $\left(\mathrm{O}_{2}^{-}\right)$directly, but electron resonance spin trapping studies suggest it (in low $\mathrm{mM}$ concentrations) may scavenge hydroxyl radical $\left(\mathrm{OH}^{\circ}\right)$. Pirfenidone forms complexes with metal ions $\left(\mathrm{eg}, \mathrm{Fe}^{2+}\right)$ that have radical scavenging activity. ${ }^{33}$ It forms a metal complex with iron when it reacts with iron chloride in ethanol and water. Pirfenidone may also modulate oxidative and nitrosative stress through inhibition of inducible nitric oxide synthase (iNOS) gene expression and decreased nitric oxide ( $\left.{ }^{\circ} \mathrm{NO}\right)$ production. That mechanism may be most relevant to the inflammatory stage of acute lungs, injury models in which $\mathrm{O}_{2}^{-}$and ${ }^{\circ} \mathrm{NO}$ react to produce peroxynitrite $\left(\mathrm{ONOO}^{-}\right)$. Peroxynitrite is widely distributed in IPF lungs and its existence may indicate previous injury of the epithelium by reactive nitrogen species. ${ }^{34}$

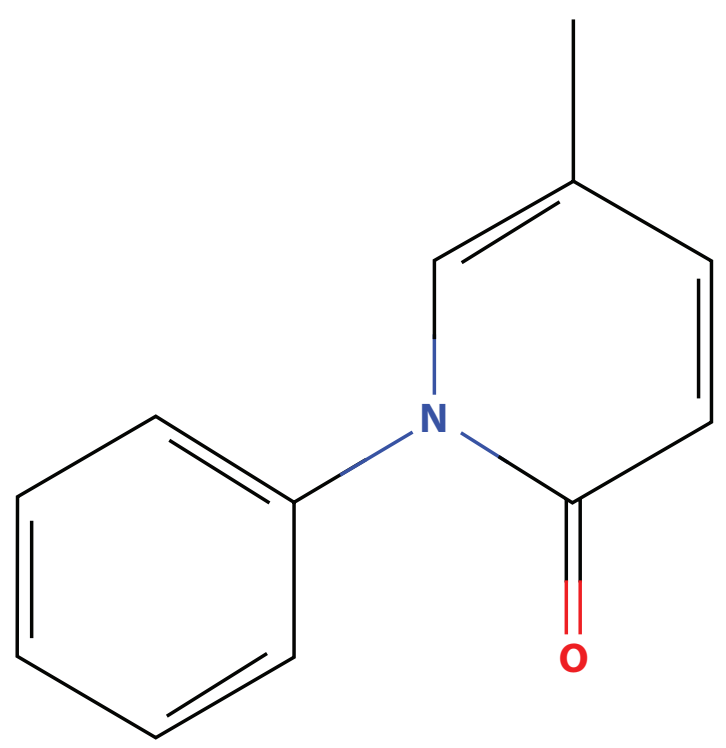

Figure 2 Diagram showing the molecular structure of pirfenidone. Pirfenidone is a heterocyclic pyridone, the molecular weight of which is $185.22 \mathrm{~g} / \mathrm{mol}$. The International Union for Physics and Applied Chemistry name for pirfenidone is 5-methyl-Iphenylpyridin-2-one. 
These properties could be related to the ability of pirfenidone to inhibit fibrosis, which is preceded by acute inflammation of the lungs in rodent models. Radical scavenging activity of pirfenidone may be less important in chronic fibrotic diseases like IPF. Pirfenidone is anti-inflammatory because it suppresses cytokine and chemokine (including TNF- $\alpha$ ) production, in addition to decreasing TGF- $\beta$, platelet derived growth factors-A and B and fibroblast growth factor.

\section{Pharmacokinetics}

Mice clear intravenous pirfenidone rapidly. The parent compound has a terminal elimination half-life of 8.6 minutes, and pirfenidone is rapidly distributed in body water. Essentially all pirfenidone is metabolized to the carboxylic acid and very little $(3 \%)$ of the parent compound is excreted intact. ${ }^{35}$

When administered orally to male and female beagles, the maximum pirfenidone concentration depended on gender. Clearance of pirfenidone (40 $\mathrm{mg} / \mathrm{kg}$ day) was $1.99 \pm 0.13$ (SE) liters/hour/kilogram and decreased with higher doses. ${ }^{36}$

After intravenous administration to sheep, organs containing the highest concentrations of pirfenidone were the lungs, liver, and intestine. Urine metabolites included hydroxylpirfenidone, carboxypirfenidone, hydroxylpirfenidoneglucuronide, and autoxypirfenidone. Less than 1\% appeared in urine as the parent drug. ${ }^{37}$

Pirfenidone's pharmacokinetics have also been studied in clinically relevant oral doses in normal human volunteers. ${ }^{5}$ Plasma levels and areas under the curve were proportional to the oral dose. Oral pirfenidone was absorbed rapidly (around 20-60 minutes) and cleared with a half life of about $2-2.5$ hours. Food intake reduced absorption, but was associated with fewer side effects.

Pharmacokinetics of pirfenidone at $801 \mathrm{mg}$ three times daily by mouth have been studied in detail in healthy subjects aged 50-79. The effects of food and antacids were assessed during the study. ${ }^{38}$ The pharmacokinetics of pirfenidone were described by a five compartment linear model with a first pass effect and first order absorption followed by a lag time. The primary metabolite, 5-carboxypirfenidone, appeared rapidly in plasma. Food reduced and delayed the absorption of pirfenidone, and it tended to increase conversion to the primary metabolite. Feeding decreased adverse reactions, including nausea and dizziness. The dose of $801 \mathrm{mg}$ three times daily with meals appeared to be appropriate and safe, as demonstrated in the clinical trials cited above.

The clinical dose of pirfenidone used for IPF therapy is $801 \mathrm{mg}$ three times daily (2403 mg total dose per day), which is reached by increasing the dose incrementally over 2 weeks. Pirfenidone is marketed as $267 \mathrm{mg}$ capsules. No dose adjustment is required in patients with mild to moderate liver disease, although liver enzymes should be monitored.

Although pirfenidone is dialyzable to a small extent, no dosage adjustment appears necessary for hemodialysis patients. ${ }^{39}$ However, pirfenidone is not recommended for use in patients with end stage renal disease or those on hemodialysis.

\section{Preclinical experiments}

Intrabroncheal instillation of bleomycin has frequently been used to model pulmonary fibrosis. Bleomycin causes an initial acute inflammatory reaction in the lung, followed by fibrosis and healing. It has been used to test potential therapies for IPF, although it may more accurately model acute lung injury and postinflammatory pulmonary fibrosis.

Bleomycin-treated hamsters develop lung fibrosis characterized by increased hydroxyproline content due to collagen and histologic evidence of diffuse alveolar damage and lung fibrosis. Feeding pirfenidone to bleomycin-instilled hamsters significantly decreased hydroxyproline and malondialdehyde, and it prevented the increase in prolyl hydroxylase activity in the lung due to bleomycin. ${ }^{4}$ Pirfenidone was also effective in decreasing lung fibrosis in a hamster model when fed after the second of three consecutive intratracheal instillations of bleomycin, ${ }^{40}$ suggesting it may be useful after the development of pulmonary fibrosis.

Pirfenidone had a similar protective effect in mice treated intraperitoneally with cyclophosphamide. Intraperitoneal cyclophosphamide causes lung fibrosis evident histologically and as increased hydroxyproline content. Ingestion of oral pirfenidone $(0.2 \% \mathrm{w} / \mathrm{w}$ in diet $)$ decreased lung hydroxyproline content but not the rate of hydroxyproline synthesis. ${ }^{41}$ These results suggest that pirfenidone may change the rate of collagen degradation or remodeling by matrix metalloproteinases.

Intratracheal bleomycin causes lung inflammation, characterized by increased inflammatory cells, protein, and TGF- $\beta$ in bronchoalveolar lavage fluid. In a hamster model of intratracheal bleomycin instillation, oral pirfenidone $(0.5 \% \mathrm{w} / \mathrm{w}$ in diet) significantly inhibited bleomycin-induced lung inflammation early after administration. ${ }^{4}$

Such studies demonstrate an effect of pirfenidone in an in vivo model of lung inflammation and fibrosis. The bleomycin model does not exactly represent IPF, but resembles acute respiratory distress syndrome (ARDS), where acute lung inflammation is followed by fibrosis that resolves with time. Extrapolation of results from the rodent 
bleomycin model of pulmonary fibrosis has not yet led to effective therapies for IPF.

Despite its limitations as a preclinical model, the bleomycin model has revealed several of pirfenidone's antifibrotic mechanisms, which involve TGF- $\beta$. Intratracheal bleomycin increases TGF- $\beta$ in bronchoalveolar lavage fluid of hamsters. Dietary pirfenidone suppresses the bleomycininduced overexpression of TGF- $\beta$ by decreasing its rate of transcription, which precedes a decrease in bronchoalveolar lavage TGF- $\beta$ protein concentration. ${ }^{4}$

Pirfenidone inhibits inflammation and cytokine production. It inhibits TNF- $\alpha$ production by a translational mechanism. ${ }^{42}$ In addition to its anti-inflammatory effect, pirfenidone down-regulates the over-expression of lung procollagen I and III genes in the hamster model. ${ }^{43}$ Pirfenidone inhibits the synthesis of PDGF-A and PDGF-B isoforms associated with a decrease in mitogenic activity of the bronchoalveolar lavage fluid. ${ }^{44}$

Pirfenidone is effective in other models of lung injury. Pirfenidone inhibits allergen-induced pulmonary inflammation in mice and guinea pigs, and it inhibits LPS-induced lung inflammation in rats. ${ }^{45}$ In these models, pirfenidone does not appear to decrease TNF- $\alpha$ in bronchoalveolar lavage fluid, but it decreases IL-6 significantly.

\section{Lung transplantation}

Pirfenidone has been tested in rodent lung transplant models in which both airway and lung fibrosis develops. Since pirfenidone inhibits TGF- $\beta$ activity and limits collagen, it has been tested and found effective in models of allograft rejection.

One of the leading causes of allograft failure is graft dysfunction caused by inflammation and progressive fibrosis. ${ }^{46}$ Pirfenidone also decreased lung myeloperoxidase activity, an indicator of inflammatory cell activation. Pirfenidone decreases TNF- $\alpha$ and appears to limit ischemia-reperfusion injury. This is a particular problem in lung transplantation, wherein chronic fibrosis of the small airways, obliterative bronchiolitis, causes graft dysfunction and failure, which may require retransplantation. Pirfenidone decreases lung collagen content in association with the down-regulation of arginase protein expression and activity attributed to a decrease in TGF- $\beta$ in the transplanted lungs. ${ }^{47}$

It is also effective in a rat model of obliterative bronchiolitis that consists of heterotopic tracheal transplantation, which histologically resembles chronic rejection. This model is not consistently reproducible and the trachea is not situated as an airway. ${ }^{48}$ It has allowed studies of immune cell function and fibrosis in rodents and it has consistently demonstrated that pirfenidone minimizes tracheal obliteration by fibrotic tissue. In a murine heterotopic tracheal transplant model, pirfenidone also inhibited the development of airway obliteration by fibrosis. ${ }^{49}$

\section{Clinical trials}

In the late 1990 s, the ability of preterminal IPF patients to tolerate pirfenidone was tested in an observational study. ${ }^{50}$ Fifty-four IPF patients received pirfenidone (up to $3600 \mathrm{mg}$ per day) for 15 days after they were perceived to fail conventional therapy (usually prednisone and an immunosuppressive). Two-year survival was 63\% (95\% confidence intervals [CI]: $50 \%-76 \%$ ), but no control group was studied, making it difficult to ascertain an effect of the drug.

A more recent trial conducted (by Azuma and colleagues) in Japan enrolled 73 pirfenidone and 36 placebo-treated subjects. ${ }^{30}$ The trial design was changed after it was underway, resulting in the exclusion of some subjects with more severe disease. The original primary endpoint, the lowest $\mathrm{O}_{2}$ saturation during a 6-minute walk test, was not significantly different, although two secondary endpoints (vital capacity and number of exacerbations) differed between the groups. The change in vital capacity after 9 months was significantly less in the pirfenidone-treated group. A data safety monitoring board stopped the trial, because of an apparent beneficial effect of pirfenidone on the number of exacerbations.

In a larger, randomized, placebo-controlled trial (by Taniguchi and colleagues) of pirfenidone in 275 IPF patients, significant differences were found in the decrease in vital capacity over 52 weeks and the progression free survival (PFS) time. The primary endpoint was the change in vital capacity and the secondary endpoint was PFS. Pirfenidone was associated with photosensitivity reactions and gastrointestinal symptoms, although it was generally well-tolerated. ${ }^{2}$ Pirfenidone significantly decreased the rate of decline in $\mathrm{VC}$ and increased the PFS time over the 52-week trial.

It is worth noting that this trial raised interesting issues regarding the choice of appropriate endpoints and the handling of missing data, which may have impacted the conclusions. ${ }^{51}$ While the decrease in the rate of deterioration of the vital capacity appeared to be favorably influenced by pirfenidone, the original endpoint was intended to be change in lowest oxygen saturation during a 6-minute walk. The authors provided ample justification for the use of vital capacity as the endpoint, although it appears that the decision to use that endpoint occurred after examination of interim data. ${ }^{51}$ The study used the last observation carried 
forward to compensate for missing data in about one-third of the subjects, and this approach may have contributed to the apparent difference between the groups. Using the last observation carried forward to replace missing data may make the group with the greater dropout rate appear to do better, if lung function decreases progressively with time. ${ }^{52}$

Although the data have not been published, two large industry sponsored randomized, placebo-controlled trials of pirfenidone in IPF have been completed. A total of 769 patients were enrolled in CAPACITY 1 and 2 trials. Pirfenidone resulted in a 30\% decrease overall in the percent of patients who experienced a $10 \%$ decrease in forced vital capacity (FVC), a primary indicator of disease progression. ${ }^{53}$ The first CAPACITY trial (PIPF-006; NCT00287729) demonstrated a favorable effect on the change in percent forced vital capacity at 72 weeks; whereas, the second CAPACITY trial (PIPF-004; NCT00287716) did not detect a significant change in percent FVC. Pooled data from the studies showed clinically important salutary effects on percent FVC, PFS, and 6-minute walk test distance. Summary data were presented to the United States Food and Drug Administration in May 2010 and are available at http:/www.fda.gov/ downloads/AdvisoryCommittees/CommitteesMeetingMate rials/Drugs/Pulmonary-AllergyDrugsAdvisoryCommittee/ UCM206399.pdf.

The primary endpoint in the CAPACITY trials and the Taniguchi study was change percent of predicted FVC at week 72 or vital capacity at week 52 , respectively. ${ }^{53}$ PFS, defined as time to death or disease progression, was a secondary endpoint. Primary and PFS endpoints were achieved in one CAPACITY trial and the Taniguchi study, but not in the second CAPACITY trial. The standardized treatment effect (STE) for change in lung function was comparable in all. Meta-analyses of the three studies showed similar STE and confidence intervals (CI), and appeared to exclude lack of effect. Hazard ratios (HR) for PFS were similar and the confidence intervals from all three studies were overlapping. HR for mortality from the meta-analysis of all three studies was 0.71 (CI: 0.57-0.89), showing a beneficial effect on survival. Results from these three Phase III studies, including meta-analyses of the STE on primary endpoints and PFS, suggested a beneficial pirfenidone effect. Pirfenidone reduced the rate of progression by $30 \%(\mathrm{HR}=0.70 ; 95 \%$ CI: 0.56-0.88).

Pirfenidone was well-tolerated by most patients. The most commonly reported adverse reactions were nausea (33\%), rash (29\%), fatigue (22\%), diarrhea (22\%), dyspepsia $(17 \%)$, and photosensitivity (12\%). All of these occurred more frequently in pirfenidone treated patients than in those receiving placebo.

These three trials provide most of the clinical information available on pirfenidone as therapy for IPF. Overall analysis of the risk of disease progression suggests that pirfenidone decreases the risk by about $30 \%$ (hazard ratio 0.7 ). ${ }^{53}$ Both the Azuma trial ${ }^{30}$ and the Taniguchi trial ${ }^{2}$ showed a decrease in the rate of decline in vital capacity favoring the pirfenidone treated groups. Additional data on pirfenidone's effect on survival time and quality of life would add certainty to its value as a clinically useful agent.

The focus of this review is the use of pirfenidone in IPF, a fibrosing lung disease that often requires lung transplantation. Pirfenidone has shown efficacy and is an active subject of research in kidney and liver diseases, among others.

Pirfenidone is effective in several experimental models of kidney fibrosis in rats. ${ }^{54-56}$ In an open label trial to assess safety and efficacy in patients with focal segmental glomerulosclerosis, the monthly change in glomerular filtration rate (GFR) was followed. The change in GFR appeared to improve in patients who received $800 \mathrm{mg}$ pirfenidone three times daily for 12 months. The study lacked a control group, and it remains possible that the improvement in GFR rate of decline may simply have reflected regression to the average rate. Only one dose of pirfenidone was tested and patients had moderate to severe chronic kidney disease at baseline, possibly biasing the study toward a negative result. ${ }^{57}$

Liver fibrosis also occurs in response to injury, and extracellular matrix accumulates as a result of transformation of cells to a myofibroblast phenotype, possibly similar to the process in fibrotic lung. ${ }^{31}$ As in the lung and kidney, several experimental models of liver fibrosis support the efficacy of pirfenidone. ${ }^{58,59}$ In a pilot study, pirfenidone (1200 mg per day for 12 months) appeared to improve liver histology in 15 patients with chronic hepatitis $\mathrm{C}$ infection. ${ }^{59}$ The histology of liver biopsies improved, as did the patients' quality of life as demonstrated by a questionnaire.

\section{Regulatory status}

Pirfenidone has been approved for use in IPF patients by Japanese and European regulatory authorities, and two large randomized controlled trials (RCT) of pirfenidone in the United States have been completed but not yet published as of early $2011 .{ }^{1}$ Four important clinical trials are summarized in Table 1.

The Japanese approval for marketing pirfenidone is based on several clinical trials including one published in 2010 . 2,30 It was placebo controlled and enrolled 275 patients; the 
Table I Summary of human trials of pirfenidone as therapy for IPF

\begin{tabular}{|c|c|c|c|c|c|}
\hline Author & Funding source & Patients enrolled & Design & Time course & Outcome measures \\
\hline Raghu $\mathrm{G}^{50}$ & $\begin{array}{l}\text { Not stated MARNAC } \\
\text { Inc provided drug }\end{array}$ & 54 & Observational & 24 months & $\begin{array}{l}\text { Survival and lung } \\
\left.\text { function (FVC, TLC, } D_{L} C O\right)\end{array}$ \\
\hline Azuma $\mathrm{A}^{30}$ & Shionogi \& Co Ltd & 109 & $\begin{array}{l}\text { Double blind, placebo } \\
\text { controlled, prospective }\end{array}$ & 9 months & $\begin{array}{l}\text { Change in lowest } \mathrm{S}_{\mathrm{P}} \mathrm{O}_{2} \text { during } \\
\text { 6-MWT VC, TLC, } D_{L} C O\end{array}$ \\
\hline Taniguchi $\mathrm{H}^{2, a}$ & $\begin{array}{l}\text { Japanese Ministry } \\
\text { of Health }\end{array}$ & 275 & $\begin{array}{l}\text { Double blind, placebo } \\
\text { controlled, prospective }\end{array}$ & 12 months & $V C^{a}$ Progression free survival \\
\hline Noble P51,b & InterMune Inc & $\begin{array}{l}779 \text { ( } 344 \text { and } 435 \text { in } \\
\text { two parallel studies) }\end{array}$ & $\begin{array}{l}\text { Double blind, placebo } \\
\text { controlled, prospective }\end{array}$ & 72 weeks & FVC Progression free survival \\
\hline
\end{tabular}

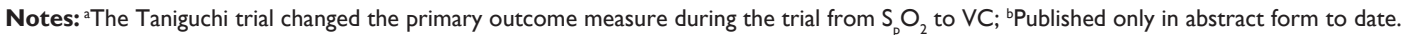

Abbreviations: 6-MWT, 6-minute walk test distance; DLCO, diffusing capacity of the lungs for carbon monoxide; FVC, forced vital capacity; IPF, idiopathic pulmonary fibrosis; $\mathrm{S}_{\mathrm{p}} \mathrm{O}_{2}$, arterial oxygen saturation measured percutaneously; TLC, total lung capacity; VC, vital capacity.

primary endpoint was the decline in vital capacity and the secondary endpoint was PFS time. Both endpoints showed positive treatment effects. The drug was well-tolerated. Photosensitivity occurred more frequently in the high (51\%) and low (53\%) dose pirfenidone treated groups, than placebo $(22 \%)$. Postmarketing efficacy and safety data for pirfenidone from Japan have not been published.

A United States Food and Drug Administration (FDA) Advisory Committee recommended approval of a pirfenidone new drug application; however the FDA declined to approve pending more data. ${ }^{1}$ A response was issued by the FDA Center for Drug Evaluation and Research which requested an additional clinical trial to confirm the effectiveness of pirfenidone. Planning for this large trial is underway. The United States distributor, InterMune, Inc, also submitted a Marketing Authorization Application to the European Medicines Agency.

Failure to achieve approval in the United States resulted from discordant results of two clinical trials in which over 700 patients were enrolled. The CAPACITY 1 and 2 trials enrolled 344 and 435 IPF patients respectively, and assessed changes in forced vital capacity at 72 weeks. Progression-free survival served as a secondary endpoint. In only one of the trials was the primary endpoint of improvement in FVC achieved.

Whether pirfenidone will be approved in the United States is not yet known. InterMune, Inc has achieved approval to market pirfenidone (as Esbriet) from the European Medicines Agency. Pirfenidone was previously granted orphan drug status for the treatment of IPF in Europe.

\section{Summary}

Pirfenidone has been investigated for many years as a potentially useful antifibrotic agent in animal models and clinical trials. However, the ultimate utility and role of pirfenidone in IPF, a fatal disease due to excess lung scarring, are yet to be settled. It clearly inhibits lung inflammation and fibrosis in the bleomycin model of lung injury and repair through mechanisms that appear to include modulating of inflammation, scavenging ROS and inhibiting fibroblast production of collagen. Pirfenidone is used as a clinical therapy to attempt to improve outcomes in IPF. Individual variability exists; however, the average effect in the overall IPF population is most important in drug development. It seems well-tolerated, but both gastrointestinal and skin adverse reactions have occurred. Results from large clinical trials have been equivocal, although unpublished data appear to show a decrease in the decline of pulmonary function and a possible survival benefit. Recent clinical trials that evaluated pirfenidone in large numbers of IPF patients have been completed but are not yet published. A preliminary meta-analysis of three pirfenidone clinical trials has been completed, and it suggests that pirfenidone may be efficacious in IPF. In addition, some proprietary data are not yet available. The ultimate role and possible utility of pirfenidone is not yet known. In the near term, it is likely that pirfenidone will prove to be a beneficial agent for patients with IPF.

\section{Disclosure}

No conflicts of interest were declared in relation to this paper.

\section{References}

1. Azuma A. Pirfenidone: antifibrotic agent for idiopathic pulmonary fibrosis. Expert Rev Resp Med. 2010;4(3):301-310.

2. Taniguchi H, Ebina M, Kondoh Y, et al. Pirfenidone in idiopathic pulmonary fibrosis. Eur Resp J. 2010;35:821-829.

3. Iyer S, Wild J, Schiedt M, et al. Dietary intake of pirfenidone ameliorates bleomycin-induced lung fibrosis in hamsters. J Labs Clin Med. 1995;125: 779-785.

4. Iyer S, Gurujeyalashmi G, Giri S. Effects of pirfenidone on transforming growth factor- $\beta$ gene expression at the transcriptional level in bleomycin hamster model of lung fibrosis. J Pharmacol Exp Therap. 1999;291: 367-373.

5. Shi S, Wu J, Chen H, Chen H, Wu J, Zeng F. Single-and multiple-dose pharmacokinetics of pirfenidone, an antifibrotic agent in healthy Chinese volunteers. J Clin Pharmacol. 2007;47:1268-1276. 
6. American Thoracic Society/European Respiratory Society International Multidisciplinary Consensus. Classification of the idiopathic interstitial pneumonias. Am J Respir Crit Care Med. 2002;165:277-304.

7. Korfei M, Ruppert C, Mahavai P, et al. Epithelial endoplasmic reticulum stress and apoptosis in sporadic idiopathic pulmonary fibrosis. Am J Repir Crit Care Med. 2008;178:838-846.

8. Raghu G, Weycker D, Edelberg J, Bradford WZ, Oster G. Incidence and prevalence of idiopathic pulmonary fibrosis. Am J Respir Crit Care Med. 2006;174:810-816.

9. Thomas AQ, Lane K, Phillips III J, et al. Heterozygosity for a surfactant protein $\mathrm{C}$ gene mutation associated with usual interstitial pneumonitis and cellular nonspecific interstitial pneumonitis in one kindred. Am J Respir Crit Care Med. 2002;165:1322-1328.

10. Baumgartner KB, Samet JM, Stidley CA, Colby TV, Waldron JA; for Division of Epidemiology and Cancer Control, University of New Mexico Health Sciences Center. Cigarette smoking: a risk factor for idiopathic pulmonary fibrosis. Am J Respir Crit Care Med. 1997;155: 242-248.

11. Taskar VS, Coultas DB. Is idiopathic pulmonary fibrosis and environmental disease? Proc Am Thorac Soc. 2006;3:293-298.

12. Raghu G, Freudenberger TD, Yang S, et al. High prevalence of abnormal acid gastro-esophageal reflux in idiopathic pulmonary fibrosis. Eur Respir J. 2006;27:136-142.

13. Wells AU, Hirani N. Interstitial lung disease guideline. Thorax. 2008; 63:v1-v58.

14. Thannickal VJ, Toews GB, White ES, Lynch III JP, Martinez FJ. Mechanisms of pulmonary fibrosis. Annu Rev Med. 2004;55:395-417.

15. Lynch DA, Godwin D, Safrin S, et al. High-resolution computed tomography in idiopathic pulmonary fibrosis. Am J Respir Crit Care Med. 2005; 172:488-493.

16. Rudd R, Harlem PL, Turner-Warwick M. Cryptogenic fibrosingalveolitis. Relationships of pulmonary physiology and bronchoalveolar lavage to response to treatment and prognosis. Am Rev Respir Dis. $1981 ; 124: 1-8$

17. Douglas WW, Ryu JH, Schroeder DR. Idiopathic pulmonary fibrosis: impact of oxygen and colchicine, prednisone, or no therapy on survival. Am J Respir Crit Care Med. 2000;161:1172-1178.

18. Turner-Warwick M, Burrows B, Johnson A. Cryptogenic fibrosingalveolitis: response to corticosteroid treatment and its effect on survival. Thorax. 1980;35:593-599.

19. Gulsvik A, Kjelsberg F, Bergmann A, Froland SS, Rootwelt K, Vale JR. High-dose intravenous methylprednisolone pulse therapy as initial treatment in cryptogenic fibrosingalveolitis. A pilot study. Respiration. 1986;50:252-257.

20. Nagai S, Kadaicha M, Ramada K, et al. Hospital-based historical cohort study of 234 histologically proven Japanese patients with IPF. Sarc Vasc Diff Lung Dis. 1999;16:209-214.

21. Selman M, Carrillo G, Salas J, et al. Colchicine, D-penicillamine, and prednisone in the treatment of idiopathic pulmonary fibrosis: a controlled clinical trial. Chest. 1998;114:507-512.

22. Johnson MA, Kwan S, Snell NJ, Nunn Al, Darbyshire JH, Turner-Warwick M. Randomized controlled trial comparing prednisolone alone with cyclophosphamide and low dose prednisolone in combination in cryptogenic fibrosingalveolitis. Thorax. 1989;44:280-288.

23. Raghu G, Depose EG, Cain K, et al. Azathioprine combined with prednisone in the treatment of idiopathic pulmonary fibrosis: a prospective double blind, randomized, placebo-controlled clinical trial. Am Rev Respir Dis. 1991;144:291-296.

24. Winterbauer RH, Hammar SP, Hallman KO, et al. Diffuse interstitial pneumonitis. Clinicopathologic correlations in 20 patients treated with prednisone/azathioprine. Am J Med. 1978;65:661-672.

25. Behera D, Gupta D, Lindal SK. Response to steroid therapy in patients of idiopathic pulmonary fibrosis: a retrospective analysis. Indian JChest Dis Allied Sci. 1998;40:163-169.

26. O’Donnell K, Keogh B, Cantin A, Crystal RG. Pharmacologic suppression of the neutrophil component of the alveolitis in idiopathic pulmonary fibrosis. Am Rev Respir Dis. 1987;136:288-292.
27. King TE, Behr J, Brown KK, et al. Build-1: randomized placebocontrolled trial of bosentan in idiopathic pulmonary fibrosis. $\mathrm{Am} \mathrm{J}$ Respir Crit Care Med. 2008;177:75-81.

28. Demedts M, Behr J, Buhl R, et al. High-dose acetylcysteine in idiopathic pulmonary fibrosis. $N$ Engl J Med. 2005;353:2229-2224.

29. Richeldi L, Davies H, Ferrara G, Franco F. Corticosteroids for idiopathic pulmonary fibrosis. Cochrane Database Syst Rev. 2003;(3):CD002880.

30. Azuma A, Nukiwa T, Tsuboi E, et al. Double-blind, placebo-controlled trial of pirfenidone in patients with idiopathic pulmonary fibrosis. Am J Respir Crit Care Med. 2005;171:1040-1047.

31. Macias-Barragan J, Sandoval-Rodriguez A, Navarro-Partida J, Armendariz-Borunda J. The multifaceted role of pirfenidone and its novel targets. Fibrogenesis Tissue Repair. 2010;3:16.

32. Misra H, Rabideau C. Pirfenidone inhibits NADPH-dependent microsomal lipid peroxidation and scavenges hydroxyl radicals. Molec Cell Biochem. 2000;204:119-126.

33. Mitani Y, Sato K, Muramoto Y, et al. Superoxide scavenging activity of pirfenidone-iron complex. Biochem Biophys Res Commun. 2008;372: 19-23.

34. Saleh D, Barnes PJ, Giaid A. Increased production of the potent oxidant peroxynitrite in the lungs of patients with idiopathic pulmonary fibrosis. Am J Respir Crit Care Med. 1997;155:1763-1769.

35. Giri S, Wang Q, Xie Y, et al. Pharmacokinetics and metabolism of a novel antifibrotic drug pirfenidone, in mice following intravenous administration. Biopharm Drug Dispos. 2002;23:203-211.

36. Bruss M, Margolin S, Giri S. Pharmacokinetics of orally administered pirfenidone in male and female beagles. $J$ Vet Pharmacol Therap. 2004;27:361-367.

37. Bruss M, Stanley S, Margolin S, Giri S. Pharmacokinetics and metabolism of intravenous pirfenidone in sheep. Biopharm Drug Dispos. 2008; 29:119-126.

38. Rubino C, Bhavnani S, Ambrose P, Forrest A, Loutit J. Effect of food and antacids on the pharmacokinetics of pirfenidone in older healthy adults. Pulm Pharmacol Ther. 2009;22:279-285.

39. Taniyama M, Ohbayashi S, Narita M, et al. Pharmacokinetics of an antifibrotic agent, pirfenidone, in hemodialysis patients. Eur J Pharmacol. 1997;52:77-78.

40. Iyer S, Margolin S, Hyde D, Giri S. Lung fibrosis is ameliorated by pirfenidone fed in diet after the second dose in a three-dose bleomycinhamster model. Exp Lung Res. 1998;24:119-132.

41. Kehrer J, Margolin J. Pirfenidone diminishes cyclophosphamideinduced lung fibrosis in mice. Toxicol Letters. 1997;90:125-132.

42. Nakazato H, Oku H, Yamane S, Tsuruta Y, Suzuki R. A novel anti-fibrotic agent pirfenidone suppresses tumor necrosis factor- $\alpha$ at the translational level. Eur J Pharmacol. 2002;446:177-185.

43. Iyer S, Gurujeyalakshmi G, Giri S. Effects of pirfenidone on procollagen gene expression at the transcriptional level in bleomycin hamster model of lung fibrosis. J Pharm Exp Therap. 1999;289:211-218.

44. Gurujeyalakshmi G, Hollinger M, Giri S. Pirfenidone inhibits PDGF isoforms in bleomycin hamster model of lung fibrosis at the translational level. Am J Physiol. 1999;276:L311-L318.

45. Spond J, Case N, Crawley C, et al. Inhibition of experimental acute pulmonary inflammation by pirfenidone. Pulmon Pharmacol Therapeutics. 2003;16:207-214

46. Liu H, Drew P, Cheng Y, Visner G. Pirfenidone inhibits inflammatory responses and ameliorates allograft injury in a rat lung transplant model. Cardiothoracic Transplant. 2005;130:852-858.

47. Liu H, Drew P, Gaugler A, Cheng Y, Visner G. Pirfenidone inhibits lung allograft fibrosis through L-arginine-arginase pathway. Am J Transplant. 2005;5:1256-1263.

48. Dosanjh A. Pirfenidone: a novel potential therapeutic agent in the management of chronic allograft rejection. Transplant Proc. 2007;39: 2153-2156.

49. Kuo E, Bharat A, Dharmarajan S, Fernandez F, Patterson G, Mohanakuma T. Animal models for bronchiolitis obliterans syndrome following human lung transplantation. Immunologic Res. 2005;33: 69-81. 
50. Raghu G, Johnson C, Lockhart D, Mageto Y. Treatment of idiopathic pulmonary fibrosis with a new antifibrotic agent, pirfenidone. Am J Resp Crit Care Med. 1999;159:1061-1069.

51. Noble P, Albera C, Bradford W, et al. Effect of pirfenidone on lung function and progression-free survival (PFS) in patients with idiopathic pulmonary fibrosis (IPF): a meta-analysis of three phase 3 studies. Am J Resp Crit Care Med. 2010;181:A1257.

52. Collard H. Idiopathic pulmonary fibrosis and pirfenidone. Eur Respir J. 2010;35:728-729.

53. Swigris J. Pirfenidone in idiopathic pulmonary fibrosis. Eur Respir J. 2010;36(3):695-696.

54. Spagnolo P, Del Giovane C, Luppi F, et al. Non-steroid agents for idiopathic pulmonary fibrosis. Cochrane Database Syst Rev. 2010;8(9): CD003134.

55. Park H, Bao L, Kim Y, et al. Pirfenidone suppressed the development of glomerulosclerosis in the FGS/Kist mouse. J Korean Med Sci. 2003; 18:527-533.
56. Giri S, Al-Bayati M, Du X, Schelegle E, Mohr F, Margolin S. Amelioration of doxorubicin induced cardiac and renal toxicity by pirfenidone in rats. Cancer Chemother Pharmacol. 2004;53:141-150.

57. Leh S, Vaagnes O, Margolin S, Iverson B, Forslund T. Pirfenidone and candesartan ameliorate morphological damage in mild chronic antiGBM nephritis in rats. Nephrol Dial Transplant. 2005;20:71-82.

58. Cho M, Smith D, Branton M, Penzak S, Kopp J. Pirfenidone slows renal function decline in patients with focal segmental glomerulosclerosis. Clin J Am Soc Nephrol. 2007;2:906-913.

59. Garcia L, Hernandez I, Sandoval A, et al. Pirfenidone effectively reverses experimental liver fibrosis. J Hepatol. 2002;37:797-805.

60. Amenariz-Borunda J, Islas-M, Meza Garcia E, et al. A pilot study in patients with established advanced liver fibrosis using pirfenidone. Gut 2006;55:1663-1665.
Transplant Research and Risk Management

\section{Publish your work in this journal}

Transplant Research and Risk Management is an international, peerreviewed open access journal focusing on all aspects of transplantation and risk management to achieve optimal outcomes in the recipient improving survival and quality of life. The journal welcomes submitted papers covering original research, basic science, clinical studies,

\section{Dovepress}

reviews \& evaluations, guidelines, expert opinion and commentary, case reports and extended reports. The manuscript management system is completely online and includes a very quick and fair peer-review system, which is all easy to use. Visit http://www.dovepress.com/ testimonials.php to read real quotes from published authors.

Submit your manuscript here: http://www.dovepress.com/transplant-research-and-risk-management-journal 